\title{
SÍNDROME DE RESPUESTA INFLAMATORIA FETAL (FIRS): ADAPTACIÓN CARDIOVASCULAR
}

\section{Fetal inflammatory response syndrome (FIRS): cardiovascular adaptation}

\author{
Mortimer Arreaza-Graterol, M.D.*, Juan Diego Rojas-Barrera, M.D.**, \\ Saulo Molina-Giraldo, M.D., M.Sc. (c)*** \\ Recibido: abril 12/10 - Aceptado: febrero 16/11
}

\section{RESUMEN}

Introducción: el síndrome de respuesta inflamatoria fetal es una condición caracterizada por reacción inflamatoria sistémica acompañada de alteraciones bioquímicas como la elevación de la interleucina 6 (IL-6). Se describió por primera vez hace una década y surgió como la necesidad de entender el comportamiento fetal en muchas situaciones clínicas conocidas. El propósito de esta revisión es brindar al lector las bases que le permitan conocer la entidad y de esta manera mejorar el oportuno diagnóstico.

Metodología: se realizó una revisión de la literatura existente de acuerdo a las bases de datos MEDLINE vía PubMed, EBSCO, Ovid y ProQuest desde el año 2000 hasta el 2009. Se

* Gineco-obstetra, Universidad El Bosque. Fellow, Medicina Materno Fetal, Hospital de San José, Fundación Universitaria de Ciencias de la Salud. Bogotá (Colombia).

** Especialista en Medicina Materno Fetal. Centro de Diagnóstico e Investigaciones Perinatales CEDIP, Universidad Católica de Chile, Hospital Sotero del Río, Unidad satélite NIH-PRB. Especialista, Unidad de Medicina Materno Fetal, Hospital San Ignacio. Docente Ginecoobstetricia, Pontificia Universidad Javeriana. Bogotá (Colombia).

*** Especialista en Medicina Materno Fetal, Hospital de San José-FUCS. Fellow, Intervención Fetal, Baylor College of Medicine, Texas Children's Fetal Center, Texas Children's Hospital. Candidato a Magíster, Docencia e Investigación Universitaria. Director, Unidad de Terapia, Cirugía Fetal y Fetoscopia, Clínica Colsubsidio Orquídeas. Especialista, Unidad de Terapia Fetal, Hospital de San José. Bogotá (Colombia). Correo electrónico: molina.saulo@ur.edu.co; saulo.molina@colsubsidio.com incluyeron artículos de revisión e investigaciones originales.

Resultados: los fetos con ruptura prematura de membranas presentan alteraciones en el llenado pasivo ventricular (E) y la contracción auricular (A). Esta relación es valorable clínicamente mediante la fórmula $\mathrm{E} / \mathrm{A}$, además de la evaluación del índice de rendimiento miocárdico, la cual es aplicable en ambos ventrículos. Los cambios en las características morfológicas de las ondas a la evaluación Doppler en fetos con ruptura prematura de membranas sugieren alta distensibilidad del ventrículo izquierdo, especialmente en fetos con infección intraamniótica. Es posible que fetos incapaces de lograr el cambio de distensibilidad cardíaca no logren mantener el volumen latido y por lo tanto no alcancen una adecuada perfusión cerebral, creándose el microambiente ideal para el desarrollo de alteraciones en el sistema nervioso central.

Conclusión: la infección e invasión microbiana de la cavidad amniótica están asociadas a cambios en la función cardiovascular fetal consistentes principalmente en un aumento de la distensibilidad ventricular.

Palabras clave: FIRS, disfunción ventricular, infección intraamniótica. 


\section{SUMMARY}

Introduction: fetal inflammatory response is a condition which is characterized by systemic inflammatory reaction accompanied by biochemical alterations such as raised interleukin 6 (IL-6) levels. It was first described over a decade ago and emerged from the need to understand fetal behavior in many known clinical situations. The purpose of this review was to provide the reader with the basis for understanding the entity and thus improve early diagnosis.

Materials and methods: a review of the pertinent literature from 2000 to 2009 was made by searching MEDLINE databases via PubMed, EBSCO, Ovid and ProQuest. Review articles and original research were included.

Results: fetuses having premature rupture of membranes (PROM) present alterations in passive ventricular filling (E) and atrial contraction (A). Such relationship is clinically evaluated by using the E/A formula and the myocardial performance index which is applicable in both ventricles. Changes in morphological characteristics regarding waves in Doppler evaluation in fetuses suffering PROM suggest high distensibility of the left ventricle, especially in fetuses exposed to intraamniotic infection. Fetuses which do not manage to change cardiac distensibility may not maintain stroke volume and thus may not achieve suitable cerebral perfusion, thereby creating an ideal microenvironment for alterations to develop in the central nervous system.

Conclusion: microbial infection and invasion of the amniotic cavity are associated with changes in fetal cardiovascular function, this being mainly consistent with increased ventricular distensibility.

Key words: FIRS, ventricular dysfunction, intraamniotic infection.

\section{INTRODUCCIÓN}

El síndrome de respuesta inflamatoria fetal (FIRS, por sus siglas en inglés) fue inicialmente descrito por Romero y colaboradores en 1997, en fetos con trabajo de parto pretérmino y ruptura prematura de membranas ovulares (PPROM). ${ }^{1}$ Es una condición caracterizada por la activación sistémica de fenómenos de inmunidad innata fetal, representada bioquímicamente por la elevación plasmática de interleucina 6 (IL-6) en plasma fetal superiores a $11 \mathrm{mg} / \mathrm{ml}^{1,2}$ La IL-6 es una glicoproteína que tiene efecto inflamatorio e induce la producción de inmunoglobulinas. ${ }^{3}$ Los fetos afectados por este síndrome presentan compromiso multiorgánico, mayor morbilidad y en caso de PPROM son más propensos a presentar parto pretérmino. La PPROM se presenta entre el 2,7 y el 17\% del total de los embarazos y la mayoría de los casos se producen espontáneamente. ${ }^{2}$ El FIRS fue propuesto como la contraparte fetal del síndrome de respuesta inflamatoria sistémica del adulto (SIRS, por sus siglas en inglés). ${ }^{2,4}$ No obstante, la definición de SIRS no puede ser aplicada al feto humano debido a que las constantes vitales, a excepción de la frecuencia cardíaca fetal, no pueden determinarse de forma expedita antes del nacimiento. Fue inicialmente descrito en casos de fetos respondiendo a la noxa infecciosa.,

El FIRS es una entidad descrita hace relativamente poco tiempo, su fisiopatología y verdadero papel todavía representan un tema de debate y un importante tópico para el enfoque de las situaciones en las cuales el feto es protagonista. ${ }^{1,2}$ Resulta entonces imprescindible para el obstetra, además del especialista en Medicina Materno Fetal, el conocimiento a cabalidad de esta sucesión de eventos y sus posibles implicaciones para así orientar las decisiones de manejo en beneficio del binomio madre-feto.

El propósito de esta revisión es describir la fisiopatología, criterios diagnósticos y consecuencias a corto y largo plazo del FIRS enfatizando en la capacidad de adaptación cardiovascular fetal durante el mismo.

\section{MATERIALES Y MÉTODOS}

Se realizó una revisión de la literatura registrada en las bases de datos MEDLINE vía PubMed, EBSCO, 
Ovid y ProQuest entre enero 1 de 2000 y diciembre 1 de 2009. Se consultaron artículos publicados en inglés, con los términos MeSH "Fetal inflammatory response syndrome", "FIRS", "fetal cardiac dysfunction", "fetal cardiovascular depression”, "chorioamnionitis", "intraamniotic infection". Por otra parte, se realizó una búsqueda adicional en la biblioteca virtual en salud LILACS y Scientific Electronic Library Online (SciELO) con el fin de establecer datos de estudios latinoamericanos pertinentes. Se incluyeron aquellos artículos de revisión, boletines informativos y protocolos con relación al tema a desarrollar. Para métodos diagnósticos, se incluyeron estudios de validez diagnóstica, y para factores de riesgo y pronóstico estudios de cohorte y casos y controles. También se incluyeron artículos clásicos en la historia de la patología. Se realizó una revisión manual, incluyendo solo aquellas publicaciones en las que se realizó el análisis del síndrome en causas, patogenia, diagnóstico y abordaje de esta entidad.

\section{RESULTADOS}

La búsqueda inicial arrojó 131 artículos. En total, de acuerdo al objetivo planteado se seleccionaron manualmente 50 artículos que cumplieron criterios de inclusión en cuanto a descripción de la entidad, fisiopatología, diagnóstico y consecuencias a corto y largo plazo desde el punto de vista cardiovascular fetal. Las bases de datos de literatura latinoamericana tales como LILACS y SciELO no arrojaron resultados.

\section{Sistema cardiovascular fetal y mediadores inflamatorios}

Teniendo en cuenta que se propone una disfunción multiorgánica fetal secundaria a una noxa cuya consecuencia es la inflamación, se hace imprescindible el análisis de los factores adaptativos desde el punto de vista cardiovascular fetal. Las alteraciones hemodinámicas secundarias a la capacidad de adaptabilidad se basan en la posibilidad de depresión miocárdica fetal luego de la reacción inflamatoria. $^{7-9}$ Son pocas las publicaciones que abordan este tema en fetos expuestos a infección intraamniótica; sin embargo, la explicación más probable para dicho efecto podría ser la acción de factores solubles, tales como productos bacterianos y citoquinas, los cuales se han encontrado elevados en la sangre del cordón de fetos infectados. ${ }^{7,9}$

Es bien conocida la existencia de factores depresores del miocardio en pacientes con SIRS o pacientes sépticos en los cuales el colapso cardíaco agudo y la incapacidad de adaptabilidad del miocardio llevan rápidamente a la muerte. Estudios recientes han reportado que la presencia de corioamnionitis histológica en neonatos se asoció con disminución de la presión media diastólica y los niveles de IL-6 se correlacionaron con las cifras de presión arterial media. ${ }^{2,10}$ Es posible que estos cambios estén presentes dentro del útero y que contribuyan en la fisiopatología de la leucomalasia periventricular y la parálisis cerebral. ${ }^{11}$ Además, en estados de sepsis fetal severa (similar al shock séptico del adulto) la depresión miocárdica puede conducir a la muerte fetal, tal como ha sido observado en casos de PPROM. ${ }^{2}$ Sin embargo, los mecanismos involucrados en la génesis de estos eventos permanecen parcialmente incomprendidos.

\section{Génesis de la infección intraamniótica y FIRS}

En años recientes, múltiples publicaciones han confirmado la asociación de la infección e inflamación intraamniótica con parto pretérmino. ${ }^{12}$ Se ha estimado que más del $40 \%$ de todos los partos pretérmino ocurren en mujeres con infección intrauterina subclínica. ${ }^{12,13}$ La invasión microbiana de la cavidad amniótica está presente en alrededor del $10 \%$ de mujeres con parto pretérmino y membranas íntegras y en 30\% de las mujeres en parto pretérmino y membranas rotas. ${ }^{12}$

La primera etapa de la infección ascendente implica el cambio y reemplazo de la flora vaginal normal del cérvix y la vagina por organismos patológicos. De forma subsecuente, ocurre la invasión y proliferación en la decidua de 
organismos patológicos, seguido del desarrollo de corioamnionitis y/o vasculitis. Finalmente, las bacterias y/o subproductos bacterianos pueden ganar acceso al feto directamente desde el líquido amniótico, donde son detectadas en asociación con otros marcadores de inflamación. ${ }^{12,13}$ Por otra parte, en la infección amniótica hay un aumento de la frecuencia de la infección materna y neonatal. La intensidad de la reacción intrauterina a la infección medida se correlaciona con los niveles de citoquinas intraamnióticas, citoquinas en sangre de cordón y con la severidad del compromiso neonatal. ${ }^{1,}{ }^{12}$ La presencia de las citoquinas incrementa la probabilidad de sepsis. $^{2,14}$

Otra forma de detección del síndrome de respuesta inflamatoria fetal es la detección de proteína $\mathrm{C}$ reactiva (PCR) en sangre de cordón umbilical, la cual se encuentra elevada en fetos con infección intraamniótica, funisitis y sepsis neonatal congénita. ${ }^{15}$ Asimismo, Samson y colegas demostraron en 1997 que los neutrófilos en el líquido amniótico son de origen fetal predominantemente. ${ }^{16}$ La evaluación histológica del cordón umbilical también es una opción para determinar si la inflamación estaba presente antes del nacimiento. La funisitis y la vasculitis coriónica son los hallazgos histopatológicos del FIRS. ${ }^{17}$ La funisitis está asociada con activación endotelial, un mecanismo clave en el desarrollo del daño orgánico. ${ }^{18}$ Finalmente, la invasión microbiana fetal puede resultar en que el síndrome de respuesta inflamatoria fetal progrese a disfunción multiorgánica, shock séptico y quizás muerte intrauterina en ausencia de finalización oportuna del embarazo. ${ }^{2}$

\section{Instauración de la respuesta inflamatoria fetal}

Durante la vida intrauterina, son varios los mecanismos que actúan para la instauración de la respuesta inflamatoria fetal. Tales mecanismos incluyen la deleción clonal de linfocitos T en el timo; la inactivación funcional y tolerancia mediada por un subgrupo de células T reguladoras; y la actividad de células funcionales reguladoras en el timo y órganos linfoides secundarios, identificada en el feto humano desde la semana 14 de edad gestacional. ${ }^{2}$

\section{Evaluación de la adaptación cardiovascular fetal}

Desde el año 2005, el índice de rendimiento miocárdico o índice de Tei, en honor a su autor, representa un método indirecto de valoración de la función cardíaca fetal de manera no invasiva y de utilidad prenatal ya establecida. ${ }^{19,20}$ El índice de funcionamiento miocárdico consiste en la relación entre la duración del período isovolumétrico (compuesto de dos períodos: contracción y relajación) y la duración del período de eyección en los ventrículos cardíacos. En el feto puede obtenerse mediante ecocardiografía utilizando el Doppler pulsado o el Doppler tisular sobre el tracto de salida del ventrículo izquierdo, obteniendo simultáneamente la morfología de la onda de entrada a nivel de la válvula mitral, y la morfología de la onda de salida a nivel aórtico; y respectivamente en el tracto de salida del ventrículo derecho a nivel de la válvula tricúspide y pulmonar; de esta forma, resulta fácil la medición del tiempo de contracción isovolumétrica, el tiempo de relajación isovolumétrica y el tiempo de eyección. ${ }^{20}$ Una función cardíaca anormal o disfunción cardíaca está asociada con prolongación del período isovolumétrico y reducción en el tiempo de eyección, todo lo cual resulta en un incremento del índice de funcionamiento miocárdico. ${ }^{21}$

Reportes recientes indican que los fetos con PPROM tienen cambios en los parámetros utilizados para evaluar la función diastólica del corazón, específicamente cambios en la onda Doppler compatibles con una gran distensibilidad del ventrículo izquierdo. ${ }^{2,7}$ Además, una reciente publicación de Letti y colegas encontró una elevación del índice de Tei en el ventrículo izquierdo de fetos con PPROM. ${ }^{19}$

Para el entendimiento de la utilización del índice de rendimiento miocárdico (índice de Tei) es fundamental considerar el análisis de la función cardíaca: la función sistólica y la función diastólica 
La función cardíaca puede estar afectada por factores independientes de la función miocárdica intrínseca, tales como la precarga y la poscarga. ${ }^{7,22}$

La disfunción diastólica ha sido reconocida como causa importante de insuficiencia cardíaca congestiva. ${ }^{7}$ La función diastólica depende de la interacción de múltiples factores como son la relajación ventricular, la succión de sangre desde las aurículas a los ventrículos, las fuerzas viscoelásticas del miocardio y la sístole auricular. ${ }^{7,23,24} \mathrm{Sin}$ embargo, los mayores determinantes del llenado ventricular son la relajación ventricular y la adecuada distensibilidad de las cámaras cardíacas. La aplicación del índice Tei al feto con FIRS permitiría la evaluación de la función ventricular de una manera no invasiva. ${ }^{7}$

La forma más común de evaluar la disfunción diastólica es mediante ecocardiografía Doppler pulsado, en la cual el volumen de muestra es colocado debajo de las válvulas aurículoventriculares. Los parámetros derivados de este tipo de evaluación no miden directamente la función diastólica ventricular, sino que miden las consecuencias hemodinámicas de la función diastólica ventricular en la morfología de ondas Doppler debajo de las válvulas aurículoventriculares. ${ }^{7}$ El período de llenado ventricular tiene dos fases: la temprana (onda $\mathrm{E}$ [early]) que depende de la relajación ventricular, y la tardía dada por la contracción auricular (onda A). ${ }^{25}$ La onda E refleja el llenado diastólico ventricular temprano, mientras que la onda A refleja cambios en la velocidad de flujo secundarios a la contracción auricular. ${ }^{7,24}$ Existe adecuada evidencia de la correlación entre la evaluación de la función diastólica mediante Doppler comparada con estudios invasivos en adultos. ${ }^{7}$

La morfología de las ondas Doppler obtenidas de la evaluación de las válvulas auriculoventriculares del feto ha sido descrita a cabalidad y son diferentes de las de neonatos, infantes y adultos. ${ }^{7,26-30}$ En condiciones normales, la onda $\mathrm{E}$ es de mayor velocidad que la A. Cuando hay dificultad de llenado ventricular, esta relación tiende a invertirse, de forma que la alteración progresiva de la función diastólica se refleja en un incremento en los valores de la relación E/A. ${ }^{25}$ Desde el punto de vista técnico, esta medición se debe realizar en un corte de 4 cámaras con una proyección apical (anterior o posterior) con un ángulo de insonación cercano a 0 grados. El volumen de muestra se coloca justo por debajo de las válvulas sigmoideas y se deben registrar al menos tres ondas similares en ausencia de movimientos respiratorios y corporales fetales. ${ }^{25}$ Se debe realizar la medición de velocidad máxima de la onda E y A y la relación entre ellas. ${ }^{25}$ La razón E/A se calcula dividiendo la velocidad pico de la onda $\mathrm{E}$ entre la velocidad pico de la onda $\mathrm{A}$, lo que refleja los cambios en la velocidad de la sangre durante la contracción auricular. En el feto, esta razón aumenta con la edad gestacional y hay predominio de la onda A durante la vida intrauterina, mientras la onda $E$ predomina en infantes y adultos. La velocidad pico y las integrales de velocidad y tiempo no cambian con la edad gestacional, mientras que en la onda E sí puede observarse un aumento en estos parámetros. Por lo tanto, los cambios en la razón de velocidad pico E/A se atribuyen a los cambios en la onda E que, como ya se mencionó, refleja el llenado ventricular temprano. ${ }^{7,30}$

Estos cambios son más notorios después de la semana 25 de gestación y han sido atribuidos al aumento de la distensibilidad cardíaca. Veille y colegas han reportado un aumento en la elasticidad del ventrículo izquierdo y disminución de la rigidez cardíaca con el avance de la gestación. ${ }^{7}$ Además, los cambios en la relajación ventricular y sus condiciones de carga pueden contribuir al aumento de la velocidad de la onda E durante el embarazo. ${ }^{7,28}$ El significado funcional de esta observación es que la función auricular es importante para alcanzar un adecuado llenado ventricular durante la vida fetal. ${ }^{7}$ La proporción velocidad pico E/A ha sido utilizada para evaluar cambios en la función diastólica ventricular y se considera que es reflejo de la distensibilidad ventricular y las condiciones de precarga. ${ }^{7}$ 
Otro parámetro para evaluar la función diastólica son las integrales de velocidad y tiempo que representan el área bajo la curva de las ondas E y A. ${ }^{7}$ Estos parámetros son proporcionales al flujo sanguíneo durante cada componente de la diástole: integral de velocidad y tiempo E (VTI E) que es proporcional al flujo sanguíneo durante el llenado ventricular temprano, integral de velocidad y tiempo A (VTI A) que es proporcional al flujo sanguíneo durante la contracción auricular, y la suma de VTI E y VTI A (VTI E y A) flujo sanguíneo total durante la diástole. Estos son los parámetros Doppler más frecuentemente utilizados para la evaluación de la función diastólica fetal. ${ }^{7}$

\section{Función diastólica en fetos con infección intraamniótica}

Los fetos con PPROM presentan una mayor razón E/A en ambos ventrículos y una mayor razón E/A de las integrales velocidad tiempo en el ventrículo izquierdo que los fetos normales. ${ }^{7}$ Los fetos con infección intraamniótica documentada presentan hallazgos similares en el ventrículo izquierdo pero no en el derecho. No se sabe con precisión la razón por la cual los hallazgos no son reproducibles en el ventrículo derecho. En adultos con shock séptico, la función del ventrículo derecho no es concordante con la del ventrículo izquierdo. ${ }^{7,8}$ Usualmente, existe un bajo volumen poscarga en el ventrículo izquierdo asociado a la hipotensión sistémica, mientras que la poscarga del ventrículo derecho se encuentra aumentada como resultado del incremento de la resistencia vascular pulmonar asociada al síndrome de distrés respiratorio del adulto. ${ }^{7,8}$ Este síndrome no existe en la vida intrauterina y la hipotensión sistémica fetal solo ocurre en casos severos previo a la muerte fetal. ${ }^{2}$ Por esta razón, han sido propuestas 3 posibles hipótesis para elucidar el compromiso predominante del ventrículo izquierdo en fetos con infección intraamniótica. Primero, la invasión microbiana del pulmón fetal puede crear una respuesta local inflamatoria y la sangre transportada del pulmón a la aurícula izquierda puede contener mayores concentraciones de factor de necrosis tumoral $\alpha$, interleucina $1 \beta$ y otros factores cardiodepresores que podrían estar ausentes en la sangre que llega a la aurícula derecha proveniente de la circulación sistémica. ${ }^{7}$ Segundo, las alteraciones en el Doppler podrían ser más fáciles de detectar debajo de la válvula mitral que debajo de la tricúspide debido a que la disminución de las ondas E y A es más frecuente en el ventrículo derecho en el feto. ${ }^{7}$ Tercero, es posible que se requiera de muestras poblacionales muy superiores a las que han sido estudiadas para detectar adecuadamente cambios en el ventrículo derecho. ${ }^{7}$ En definitiva, si se considera a la relación E/A como un reflejo de la función diastólica, las observaciones de Romero y colegas sugieren que los fetos con PPROM tienen cambios en la función diastólica cuando se les compara con fetos normales. ${ }^{7}$

Los cambios en las características morfológicas de las ondas a la evaluación Doppler en fetos con PPROM sugieren una alta distensibilidad del ventrículo izquierdo, especialmente en fetos con infección intraamniótica probada. ${ }^{2,7}$ Es coincidente con esta interpretación el hecho de que un mayor grado de llenado ventricular izquierdo ocurre durante la diástole temprana en fetos infectados que en aquellos sanos. Esta variación puede atribuirse a un menor llenado ventricular durante la sístole auricular. ${ }^{7}$ Durante los primeros días del shock séptico, el patrón de depresión miocárdica en adultos se caracteriza por dilatación del ventrículo izquierdo, disminución de la fracción de eyección ventricular y un índice cardíaco normal o elevado. ${ }^{7,31}$ En pacientes que sobreviven el evento es frecuente observar dilatación ventricular aguda en los primeros días. Esto ha sido atribuido a una dilatación ventricular compensatoria que ocurre para mantener el volumen latido a pesar de la pérdida importante de la contractilidad miocárdica. ${ }^{7,31}$ Estudios recientes han propuesto que los cambios en la función cardíaca diastólica observados en los fetos con infección intraamniótica representan un 
mecanismo compensatorio similar al de adultos con sepsis. ${ }^{7}$ Es posible que aquellos fetos incapaces de lograr el cambio de distensibilidad cardíaca no logren mantener el volumen latido y el trabajo cardíaco y por lo tanto no alcancen una adecuada perfusión cerebral, resultando predispuestos a presentar hipotensión e isquemia cerebral en el útero, creándose el microambiente ideal para el desarrollo de la leucomalasia periventricular. ${ }^{7}$ Por lo tanto, los cambios en la función diastólica reportados en la literatura que presentan los fetos con infección intraamniótica podrían tener valor protector e incluso de sobrevivencia.

La razón por la cual fetos sin evidencia de invasión microbiana de la cavidad amniótica también presentan cambios en la función diastólica es incierta. Una posible explicación de este fenómeno observado en fetos con PPROM es la ocurrencia de inflamación intraamniótica, donde aún en presencia de un cultivo negativo puede ocurrir una reacción inflamatoria. ${ }^{7,32}$

\section{Etiopatogenia de la disfunción cardiovascular}

Los lipopolisacáridos bacterianos (LPS) o endotoxinas, componentes de la pared celular de bacterias gram-negativas, han sido implicados en la fisiopatología del parto pretérmino, ${ }^{33,34} \mathrm{PPROM}^{35}$ y shock séptico. ${ }^{36}$ El mecanismo mediante el cual los LPS inducen esta respuesta celular ha sido definido parcialmente. Los LPS se unen a una proteína reactante de fase aguda conocida como proteína asociadora de lipopolisacáridos (LPB) formando un complejo LPS-LPB que a su vez se une al receptor CD14, un receptor presente en la superficie de neutrófilos, macrófagos y monocitos. ${ }^{37} \mathrm{La}$ interacción del receptor CD14 con el complejo LPS-LPB inicia la traducción de señales con la participación del receptor TLR4. ${ }^{38}$ Esto eventualmente lleva a la activación de la proteincinasa activada por mitógenos y la vía del factor nuclear $\kappa \mathrm{B} .{ }^{39}$ Estos eventos resultan en la producción de citoquinas proinflamatorias ya mencionadas en este escrito como son el factor de necrosis tumoral $\alpha$ y la interleucina $1 .^{40}$

La patogénesis de la disfunción miocárdica se fundamenta en las siguientes observaciones: 1) las concentraciones plasmáticas del factor de necrosis tumoral $\alpha$ se encuentran aumentadas en adultos y niños con sepsis; ${ }^{7,41}$ 2) ratones transgénicos modificados para producir factor de necrosis tumoral $\alpha$ miocárdico desarrollaron cardiomiopatía dilatada caracterizada por dilatación ventricular y disminución de la fracción de eyección;,42 3) la administración de factor de necrosis tumoral $\alpha$ a animales produce depresión miocárdica dosis dependiente; ${ }^{7,43}$ 4) la incubación de tejido miocárdico, humano y animal, con factor de necrosis tumoral alfa resulta en depresión de la contractilidad dosis dependiente; ${ }^{7,44}$ 5) el factor de necrosis tumoral alfa induce apoptosis en miocitos cardíacos; $;^{7,45}$ 6) el factor inhibitorio de macrófagos, que se encuentra elevado en la invasión microbiana de la cavidad amniótica, tiene efectos cardiodepresores. ${ }^{7,46,47}$

\section{Implicaciones clínicas}

Las pruebas de vigilancia utilizadas actualmente para la monitorización de pacientes con PPROM son principalmente la monitorización fetal y el perfil biofísico. Estas son herramientas útiles para la detección del feto con mayor riesgo de infección y para planificar el momento óptimo del parto. Lamentablemente, son pruebas insuficientes para caracterizar la respuesta cardiovascular fetal a fenómenos de infección/inflamación. ${ }^{7}$

Es posible que el futuro de la vigilancia fetal en pacientes con posible infección/inflamación de la cavidad amniótica incluya parámetros de función cardiovascular, particularmente en fetos con edades gestacionales muy lejanas al término, en quienes una decisión de finalización del embarazo pueda comprometer la supervivencia.?

Recientemente, se han utilizado anticuerpos monoclonales contra factor de necrosis tumoral alfa para prevenir la dilatación ventricular y la 
disminución de la contractilidad miocárdica inducida por polisacáridos bacterianos en modelos animales de sepsis. ${ }^{7,48}$ Estudios clínicos con un anticuerpo monoclonal anti factor de necrosis tumoral $\alpha$ en pacientes con shock séptico han reportado mejoría de la función del ventrículo izquierdo sin disminuir la mortalidad del grupo de pacientes. ${ }^{7,49}$

Dado que algunos neonatos presentan elevados niveles de factor de necrosis tumoral $\alpha$ al momento del nacimiento, podría plantearse el uso de moduladores de respuesta inmune innata (anti TNF $\alpha$, interleucina 10 y anti factor inhibitorio de macrófagos) para la sepsis neonatal de inicio temprano., ${ }^{7,50}$

\section{DISCUSIÓN}

El síndrome de respuesta inflamatoria fetal está presente en aproximadamente la mitad de los casos de PPROM. Asumiendo el peor escenario donde al menos 1 de cada 2 fetos en este contexto se encuentra infectado o potencialmente infectado, resulta de importancia capital conocer las implicaciones del síndrome de respuesta inflamatoria fetal en todos los órganos involucrados con el fin de entender los cambios fisiológicos de adaptabilidad fetal que hasta el momento de la descripción de la entidad parecían desconocidos.

La disfunción cardiovascular fetal y neonatal en el escenario del FIRS ha sido evaluada a cabalidad por el grupo de Romero y colegas, y el grupo de Yanowitz y colegas y los hallazgos asociados a esta, han sido descritos a lo largo de esta revisión. Dentro del contexto de la disfunción multiorgánica fetal presente en el FIRS en la infección intrauterina, hemos descrito que existen factores medibles que permiten objetivizar la valoración funcional cardíaca del feto mediante la técnica de la ecocardiografía, por lo cual consideramos obligatorio el entendimiento de los principales factores fisiopatológicos de adaptación hemodinámica con el fin de utilizar la herramienta diagnóstica. Además de darnos una importante información acerca de las alteraciones morfológicas, representa un método importantísimo desde el punto de vista funcional, la valoración de las características miocárdicas y hemodinámicas fetales adaptativas. Es de anotar que el ecocardiograma fetal debe ser realizado en Unidades Materno Fetales de experiencia, las cuales deben contar con el recurso humano necesario para ofrecer a nuestras pacientes la oportunidad de un diagnóstico adecuado que permita identificar situaciones en las cuales la vida intrauterina se vea comprometida.

Las observaciones de los grupos de investigación antes mencionados, en opinión de los autores, dejan la puerta abierta para la potencial realización de diversos estudios cardiovasculares no invasivos que faciliten la consecución del diagnóstico de FIRS de forma expedita, disminuyendo la morbi-mortalidad neonatal asociada a este síndrome. Llama la atención cómo, dentro de la revisión realizada, las bases de datos latinoamericanas consultadas no arrojaron publicaciones que cumplieran criterios de inclusión; sin embargo es del conocimiento de los autores que grupos de investigación de Chile y Brasil han ahondado en el tema y sus escritos se encuentran en publicaciones anglosajonas de mayor impacto. Nuestro país no puede ser ajeno a la búsqueda de líneas de investigación que admitan la aplicabilidad de los conceptos revisados en el diseño de estudios locales que permitan el mejor entendimiento del papel de feto en problemas como la infección intrauterina, que son frecuentes en nuestro medio y que todavía representan un grupo de entidades que aumentan la morbimortalidad materno fetal en la población vulnerable.

La debilidad de nuestra revisión radica en ser no sistemática, lo cual produciría un sesgo en la selección de los artículos; sin embargo, los objetivos iniciales de dar a conocer los hallazgos en cuanto a fisiopatología, diagnóstico, importancia de la entidad y, sobre todo, la descripción de cambios adaptativos cardiovasculares son introducidos de una manera práctica. 


\section{CONCLUSIÓN}

El síndrome de respuesta inflamatoria fetal (FIRS) es una entidad cuyo conocimiento reviste particular importancia en el abordaje del feto cuya madre cursa con infección intraamniótica con o sin membranas rotas. La infección e invasión microbiana de la cavidad amniótica están asociadas a cambios en la función cardiovascular fetal, consistentes principalmente en aumento de la distensibilidad ventricular los cuales son objetivamente valorables mediante un abordaje completo y oportuno por parte de una Unidad Materno Fetal con experiencia. El conocimiento de esta entidad permitirá entender los mecanismos de adaptabilidad fetal a diferentes entidades inflamatorias, lo cual permitirá el mejor abordaje clínico en casos específicos.

\section{REFERENCIAS}

1. Gómez R, Romero R, Ghezzi F, Yoon BH, Mazor M, Berry SM. The fetal inflammatory response syndrome. Am J Obstet Gynecol 1998;179:194-202.

2. Gotsch F, Romero R, Kusanovic J, Mazaki-Tovi S, Pineles BL, Erez O, et al. The fetal inflammatory response syndrome. Clin Obstet Gynecol 2007;50:652-83.

3. Moser B, Willimann K. Chemokines: role in inflammation and immune surveillance. Ann Rheum Dis 2004;63:ii84-ii89.

4. American College of Chest Physicians/Society of Critical Care Medicine Consensus Conference. Definitions for sepsis and organ failure and guidelines for the use of innovative therapies in sepsis. Crit Care Med 1992;20:864-74.

5. Riedemann NC, Guo RF, Ward PA. Novel strategies for the treatment of sepsis. Nat Med 2003;9:517-24.

6. Cotton DB, Hill LM, Strassner HT, Platt LD, Ledger WJ. Use of amniocentesis in preterm gestation with ruptured membranes. Obstet Gynecol 1984;63:38-48.

7. Romero R, Espinoza J, Gonçalves LF, Gomez R, Medina L, Silva M, et al. Fetal cardiac dysfunction in preterm premature rupture of membranes. J Matern Fetal Neonatal Med 2004;16:146-57.

8. Kumar A, Haery C, Parrillo JE. Myocardial dysfunction in septic shock: Part I. Clinical manifestation of cardiovascular dysfunction. J Cardiothorac Vasc Anesth 2001;15:364-76.
9. Court O, Kumar A, Parrillo JE, Kumar A. Clinical review: myocardial depression in sepsis and septic shock. Crit Care 2002;6:500-8.

10. Yanowitz TD, Jordan JA, Gilmour CH, Towbin R, Bowen A, Roberts JM, et al. Hemodynamic disturbances in premature infants born after chorioamnionitis: association with cord blood cytokine concentrations. Pediatr Res 2002;51:310-6.

11. Garnier Y, Coumans AB, Jensen A, Hasaart TH, Berger R. Infection-related perinatal brain injury: the pathogenic role of impaired fetal cardiovascular control. J Soc Gynecol Investig 2003;10:450-9.

12. Arad I, Ergaz Z. The fetal inflammatory response syndrome and associated infant morbidity. Isr Med Assoc J 2004;6:766-9.

13. Romero R, Chaiworapongsa T, Espinoza J. Micronutrients and intrauterine infection, preterm birth and the fetal inflammatory response syndrome. J Nutr 2003;133:1668S-1673S.

14. Yoon BH, Romero R, Kim KS, Park JS, Ki SH, Kim BI, et al. A systemic fetal inflammatory response and the development of bronchopulmonary dysplasia. Am J Obstet Gynecol 1999;181:773-9.

15. Yoon BH, Romero R, Shim JY, Shim SS, Kim CJ, Jun JK. C-reactive protein in umbilical cord blood: a simple and widely available clinical method to assess the risk of amniotic fluid infection and funisitis. J Matern Fetal Neonatal Med 2003;14:85-90.

16. Sampson JE, Theve RP, Blatman RN, Shipp TD, Bianchi DW, Ward BE, et al. Fetal origin of amniotic fluid polymorphonuclear leukocytes. Am J Obstet Gynecol 1997;176:77-81.

17. Pacora P, Chaiworapongsa T, Maymon E, Kim YM, Gomez R, Yoon BH, et al. Funisitis and chorionic vasculitis: the histological counterpart of the fetal inflammatory response syndrome. J Matern Fetal Neonatal Med 2002;11:18-25.

18. D'Alquen D, Kramer BW, Seidenspinner S, Marx A, Berg D, Groneck P, et al. Activation of umbilical cord endothelial cells and fetal inflammatory response in preterm infants with chorioamnionitis and funisitis. Pediatr Res 2005;57:263-9.

19. Letti Müller AL, Barrios Pde M, Kliemann LM, Valério EG, Gasnier R, Magalhães JA. Tei index to assess fetal cardiac performance in fetuses at risk for fetal inflammatory response syndrome. Ultrasound Obstet Gynecol 2010;36:26-31. 
20. Hernández-Andrade E, López-Tenorio J, FigueroaDiesel H, Sanin-Blair J, Carreras E, Cabero L, et al. A modified myocardial performance (Tei) index based on the use of valve clicks improves reproducibility of fetal left cardiac function assessment. Ultrasound Obstet Gynecol 2005;26:227-32.

21. Wolfberg AJ, Norwitz EL. Probing the fetal cardiac signal for antecedents of brain injury. Clin Perinatol 2009;36:673-84.

22. Ross JJ. Assessment of cardiac function and myocardial contractility. En: Schlant RC, Alexander RW, O'Rourke RA, et al. eds. The Heart. New York: McGraw-Hill; 1994. p. 487-502.

23. Naqvi TZ. Diastolic function assessment incorporating new techniques in Doppler echocardiography. Rev Cardiovasc Med 2003;4:81-99.

24. Nishimura RA, Tajik AJ. Evaluation of diastolic filling of left ventricle in health and disease: Doppler echocardiography is the clinician's Rosetta Stone. J Am Coll Cardiol 1997;30:8-18.

25. Hernández-Andrade E, Figueroa-Diesel H, Kottman C, Illanes S, Arraztoa J, Acosta-Rojas R, et al. Gestational-age-adjusted reference values for the modified myocardial performance index for evaluation of fetal left cardiac function. Ultrasound Obstet Gynecol 2007;29:321-5.

26. Carceller-Blanchard AM, Fouron JC. Determinants of the Doppler flow velocity profile through the mitral valve of the human fetus. Br Heart J 1993;70:457-60

27. Chang CH, Chang FM, Yu CH, Liang RI, Ko HC, Chen HY. Systemic assessment of fetal hemodynamics by Doppler ultrasound. Ultrasound Med Biol 2000;26:777-85.

28. Fernández Pineda L, Tamariz-Martel Moreno A, Maître Azcárate MJ, López Zea M, Rico Gómez F, Cazzaniga Bullón M, et al. Contribution of Doppler atrioventricular flow waves to ventricular filling in the human fetus. Pediatr Cardiol 2000;21:422-8.

29. Gardiner H, Brodszki J, Marsal K. Ventriculovascular physiology of the growth-restricted fetus. Ultrasound Obstet Gynecol 2001;18:47-53.

30. Harada K, Rice MJ, Shiota T, Ishii M, McDonald RW, Reller MD, et al. Gestational age- and growth-related alterations in fetal right and left ventricular diastolic filling patterns. Am J Cardiol 1997;79:173-7.

31. Parker MM, Shelhamer JH, Bacharach SL, Green MV, Natanson C, Frederick TM, et al. Profound but reversible myocardial depression in patients with septic shock. Ann Intern Med 1984;100:483-90.

32. Shim SS, Yoon BH, Romero R, Hong JS, Kim G, Sohn $\mathrm{YK}$, et al. The frequency and clinical significance of intra-amniotic inflammation in patients with preterm premature rupture of the membranes. Am J Obstet Gynecol 2003;189:S83.

33. Romero R, Sirtori M, Oyarzun E, Avila C, Mazor M, Callahan R, et al. Infection and labor. V. Prevalence, microbiology, and clinical significance of intraamniotic infection in women with preterm labor and intact membranes. Am J Obstet Gynecol 1989;161:817-24.

34. Fidel PI Jr, Romero R, Maymon E, Hertelendy F. Bacteria-induced or bacterial product-induced preterm parturition in mice and rabbits is preceded by a significant fall in serum progesterone concentrations. J Matern Fetal Med 1998;7:222-6.

35. Romero R, Roslansky P, Oyarzun E, Wan M, Emamian M, Novitsky TJ, et al. Labor and infection. II. Bacterial endotoxin in amniotic fluid and its relationship to the onset of preterm labor. Am J Obstet Gynecol 1988;158:1044-9.

36. Van Amersfoort ES, Van Berkel TJ, Kuiper J. Receptors, mediators, and mechanisms involved in bacterial sepsis and septic shock. Clin Microbiol Rev 2003;16:379414.

37. Schumann RR, Zweigner J. A novel acute-phase marker: lipopolysaccharide binding protein (LBP). Clin Chem Lab Med 1999;37:271-4.

38. Hoshino K, Takeuchi O, Kawai T, Sanjo H, Ogawa T, Takeda Y, et al. Cutting edge: Toll-like receptor 4 (TLR4)-deficient mice are hyporesponsive to lipopolysaccharide: evidence for TLR4 as the Lps gene product. J Immunol 1999;162:3749-52.

39. Downey JS, Han J. Cellular activation mechanisms in septic shock. Front Biosci 1998;3:d468-76.

40. Fenton MJ, Golenbock DT. LPS-binding proteins and receptors. J Leukoc Biol 1998;64:25-32.

41. Casey LC, Balk RA, Bone RC. Plasma cytokine and endotoxin levels correlate with survival in patients with the sepsis syndrome. Ann Intern Med 1993;119:771-8.

42. Bryant D, Becker L, Richardson J, Shelton J, Franco F, Peshock R, et al. Cardiac failure in transgenic mice with myocardial expression of tumor necrosis factoralpha. Circulation 1998;97:1375-81.

43. Eichenholz PW, Eichacker PQ, Hoffman WD, Banks SM, Parrillo JE, Danner RL, et al. Tumor necrosis 
factor challenges in canines: patterns of cardiovascular dysfunction. Am J Physiol 1992;263:H668-75.

44. Goldhaber JI, Kim KH, Natterson PD, Lawrence T, Yang P, Weiss JN. Effects of TNF-alpha on [Ca2 + ] $i$ and contractility in isolated adult rabbit ventricular myocytes. Am J Physiol 1996;271:H1449-55.

45. Krown KA, Page MT, Nguyen C, Zechner D, Gutierrez V, Comstock KL, et al. Tumor necrosis factor alphainduced apoptosis in cardiac myocytes. Involvement of the sphingolipid signaling cascade in cardiac cell death. J Clin Invest 1996;98:2854-65.

46. Garner LB, Willis MS, Carlson DL, DiMaio JM, White MD, White DJ, et al. Macrophage migration inhibitory factor is a cardiac-derived myocardial depressant factor. Am J Physiol Heart Circ Physiol 2003;285:H2500-9.

47. Chaiworapongsa T, Romero R, Espinoza J, Kim YM, Edwin S, Bujold E, et al. A novel mediator of septic shock, macrophage migration inhibitory factor, is increased in intra-amniotic infection. Am J Obstet Gynecol 2002;187:S73.

48. Kraut EJ, Chen S, Hubbard NE, Erickson KL, Wisner DH. Tumor necrosis factor depresses myocardial contractility in endotoxemic swine. J Trauma 1999;46:900-6.

49. Abraham E, Anzueto A, Gutierrez G, Tessler S, San Pedro G, Wunderink R, et al. Double-blind randomised controlled trial of monoclonal antibody to human tumour necrosis factor in treatment of septic shock. NORASEPT II Study Group. Lancet 1998;351:929-33.

50. Rounioja S, Räsänen J, Glumoff V, Ojaniemi M, Mäkikallio K, Hallman M. Intra-amniotic lipopolysaccharide leads to fetal cardiac dysfunction. A mouse model for fetal inflammatory response. Cardiovasc Res 2003;60:156-64. 